\title{
Vibrational scaling of the heterogeneous dynamics detected by mutual information
}

\author{
Antonio Tripodo ${ }^{1}$, Francesco Puosi $^{1}$, Marco Malvaldi $^{1}$, and Dino Leporini ${ }^{1,2}$ a \\ 1 Dipartimento di Fisica “Enrico Fermi”, Università di Pisa, Largo B.Pontecorvo 3, I-56127 Pisa, Italy \\ 2 IPCF-CNR, UOS Pisa, Italy
}

Received: date / Revised version: date

\begin{abstract}
The correlations detected by the mutual information in the propensities of a molecular viscous liquid are studied by molecular-dynamics simulations. Dynamic heterogeneity is evidenced and two particle fractions with different mobility and relaxation identified. The two fractions exhibit scaling of their relaxation in terms of the rattling amplitude of the particle trapped in the cage of the first neighbours $\left\langle u^{2}\right\rangle$. The scaling master curve does not differ from the one found for bulk systems, thus confirming identical results previously reported in other systems with strong dynamic heterogeneity as thin molecular films. Excitation of planar and globular structures at short and long times with respect to structural relaxation, respectively, is revealed. Some of the globular structures are different from the ones evidenced in atomic mixtures. States with equal $\left\langle u^{2}\right\rangle$ are found to have identical time dependence of several quantities, referring to both bulk and the two fractions with heterogeneous dynamics, at least up to the structural relaxation time $\tau_{\alpha}$.
\end{abstract}

PACS. XX.XX.XX No PACS code given

\section{Introduction}

The nature of the progressive solidification of a liquid to get to the amorphous glassy state is a major scientific challenge [1-5]. On approaching the glass transition, trapping effects due to the cage formed by the first neighbors are more and more prominent and the average escape time, i.e. the structural relaxation time $\tau_{\alpha}$, increases from a few picoseconds up to thousands of seconds. The caged particles are not completely immobilized by the surroundings but they wiggle with mean-square amplitude $\left\langle u^{2}\right\rangle$ on the picosecond time scale $t^{\star}$. Henceforth, $\left\langle u^{2}\right\rangle$ which is strictly related to the the Debye-Waller factor, will be referred to as fast mobility (FM).

Despite the huge range of time scales earlier [6] and later theoretical [7-13] and experimental [14] studies addressed the rattling process within the cage to understand the structural relaxation rising a growing interest on the FM role [15-37]. In particular, correlations between FM and structural relaxation are found in polymers in bulk [20-22,36] and thin films [37], binary atomic mixtures [21,30], colloidal gels [25], antiplasticized polymers [29, 38], water [32] and water-like models $[34,35]$. FM also provided an alternative interpretation of the so-called thermodynamic (or temperature/density) scaling [33]. The correlation between structural relaxation and FM has been inspected in the experimental data concerning several glassformers in a wide range of fragility - the steepness index $m$ defined by Angell [39] - (20 $\leq m \leq 191)$, including polymers, van der Waals and hydrogen-bonded liquids, metallic

\footnotetext{
${ }^{a}$ e-mail: dino.leporinieunipi.it
}

glasses, molten salts and the strongest inorganic glassformers $[20,23,24,30-33]$. The correlation is summarized by the universal master curve [20]:

$$
\begin{aligned}
\log \tau_{\alpha} & =\mathcal{F}_{F M}\left(\left\langle u^{2}\right\rangle\right) \\
& =\tilde{\alpha}+\tilde{\beta}\left(\frac{\left\langle u_{g}^{2}\right\rangle}{\left\langle u^{2}\right\rangle}\right)+\tilde{\gamma}\left(\frac{\left\langle u_{g}^{2}\right\rangle}{\left\langle u^{2}\right\rangle}\right)^{2}
\end{aligned}
$$

$\left\langle u_{g}^{2}\right\rangle$ is the FM at the glass transition, $\tilde{\beta}$ and $\tilde{\gamma}$ are systemindependent constants and $\tilde{\alpha}=2-\tilde{\beta}-\tilde{\gamma}$ to comply with the usual definition $\tau_{\alpha}=100 \mathrm{~s}$ at the glass transition. Douglas and coworkers developed a localization model predicting the alternative master curve $\mathcal{F}_{F M}\left(\left\langle u^{2}\right\rangle\right) \propto\left\langle u^{2}\right\rangle^{-3 / 2}[29,38,40]$.

Further studies revealed that the master curve, Eq.1, is a manifestation of a more intimate correlation between the vibrational dynamics and the slow relaxation, i.e., if two states have equal FM, they have coincident time evolution of any ensemble-averaged quantity $X(t)$ at least between $t^{\star}$ and $\tau_{\alpha}$ [22]. Said otherwise, for $t^{*} \lesssim t \lesssim \tau_{\alpha}$ at least, it holds

$$
\left\langle u^{2}\right\rangle_{(1)}=\left\langle u^{2}\right\rangle_{(2)} \Rightarrow X(t)_{(1)}=X(t)_{(2)}
$$

In selected systems Eq.3 extends up to even longer times, e.g. atomic binary mixtures and unentangled polymers, where the diffusive regime is covered as well [22, 26, 30, 36]. Eq.3 has been tested for the intermediate scattering functions, mean square displacement and non-gaussian parameter [20, 23, 24, 30], van Hove function [22], transient elasticity [41] and displacementdisplacement correlation functions $[27,28]$. 
Following first indications [20], the role of the vibrational dynamics has been noted in systems with heterogeneous $d y$ namics - e.g. glassformers close to the glass transition [26$28,36]$ where the Stokes-Einstein relation fails $[26,36]$ and thin films with strong mobility gradient [37] - where distinct FM is found in regions with different relaxation and transport at long times. Insight into the structural origin of the longtime dynamic heterogeneity has been provided by propensity, the random particle displacement in times longer than the vibrational ones starting from the same initial global configuration, thus forming the so-called 'iso-configurational ensemble' (ICE) $[17,19,42]$.

Motivated by the previous remarks, the present paper focuses on the dynamic heterogeneity of dense liquids where it has been revealed that more and more particles move in a correlated way approaching the glass transition, e.g. see the review in ref. [5]. In particular, we investigate if: i) both the master curve Eq. 2 and Eq. 3 hold in the regions with different FM and relaxation and ii) there are structural signatures, especially at short times $t \sim t^{*}$, of the long-time dynamic heterogeneity. As to the latter aspect, we profit from previous studies on the local geometry and collective extended excitations driving the moves of a particle in the cage of its neighbours in dense liquids $[15,43]$.

We examine the dynamic correlations detected by mutual information (MI). MI between two random variables is a measure of their, possibly nonlinear, statistical dependence (correlation) [44]. MI between the random vector variables $\mathbf{X}$ and $\mathbf{Y}$ is defined as [45]:

$$
I(\mathbf{X}, \mathbf{Y})=\iint d \mathbf{x} d \mathbf{y} p(\mathbf{x}, \mathbf{y}) \log \left[\frac{p(\mathbf{x}, \mathbf{y})}{p(\mathbf{x}) p(\mathbf{y})}\right]
$$

where $p(\mathbf{x}, \mathbf{y})$ is the joint probability distribution of the variables $\mathbf{X}$ and $\mathbf{Y}$ with marginal distributions $p(\mathbf{x})$ and $p(\mathbf{y})$, respectively. MI has been used for detecting classical phase transitions for several classical system and topological transition in the XY model [46], the phase transition in a 2D disordered Ising model [47] and for evaluating the configurational entropy of liquid metals [48]. In the framework of liquid-state physics MI has been considered in atomic glassformers as a metric of the correlation between structural and dynamical quantities $[45,49,50]$, in particular the propensity $[45,50]$. Since MI revealed the presence of subsets of particles with different relaxation properties in molecular-dynamics (MD) simulations of an atomic liquid mixture [45], it offers the opportunity to see if these subsets comply with the vibrational scaling as expressed by Eq.2 and Eq.3. More specifically, we investigate by MD simulations the MI correlations in space and time of the propensities of a model molecular glassformer with the same scheme of ref. [45]. We design our molecular liquid to limit the differences with atomic liquids in an attempt to test the sensitivity of MI correlations. To this aim, we consider short linear trimers where bending and torsional potentials are missing to ensure the highest molecular flexibility and constrain the monomer displacements as less as possible. The bond length was taken slightly shorter than the equilibrium point of the Lennard-Jones (LJ) non-bonding potential to avoid crystallization issues while ensuring limited perturbation of the local order.
The paper is organized as follows. In Sec. 2 details about the numerical models and methods are given. The results are presented and discussed in Sec. 3.

\section{Models and methods}

We performed MD simulations of fully-flexible, i.e. with no bond-bond bending potential, linear chains of trimers in the mildly supercooled regime. All simulations were carried out with the open-source software LAMMPS [51,52]. Non-bonded monomers interact with a truncated LJ potential

$$
U^{L J}(r)=\epsilon\left[\left(\frac{\sigma^{*}}{r}\right)^{12}-2\left(\frac{\sigma^{*}}{r}\right)^{6}\right]+U_{\text {cut }}(r)
$$

where $\sigma^{*}=2^{1 / 6} \sigma$ is the location of the potential minimum which has depth $\epsilon$. $U_{\text {cut }}$ is chosen to ensure $U^{L J}(r)=0$ for any $r>r_{c u t}$ and $r_{c u t}$ is set to the value of $2.5 \sigma$. Monomers that belongs to the same chain interact with each other via the harmonic potential $U^{b}(r)=k\left(r-r_{0}\right)^{2}$ where the constant $k$ is set to $555.5 \mathrm{\epsilon} / \sigma^{2}$ and the rest length of the bond is $r_{0}=0.97 \sigma$. All quantities are in reduced units: length in units of $\sigma$, temperature in units of $\epsilon / k_{B}$ and time in units of $\sigma \sqrt{m / \epsilon}$ where $m$ is the monomer mass. We set $m=k_{B}=1$. The time step for the integration was chosen to be 0.003 . All the investigated systems have $N=3999$ monomers, i.e. 1333 chains. The NVT ensemble has been used for the equilibration runs. For each run the equilibration time lasted not less than $3 \tau_{e e}$, where $\tau_{e e}$ is the end to end vector autocorrelation function decay time [53-62].

After equilibration, the NVE ensemble was employed for the conventional data production employing at least sixteen independent runs. We built three sets of states varying both the density $\rho$ and temperature $T$, each state being characterized by the pair $(\rho, T)$ : set $A=\left\{A_{1}, A_{2}\right\}$ with $A_{1}=(1.05,0.60)$, $\mathrm{A}_{2}=(1.01,0.47)$; set $\mathrm{B}=\left\{\mathrm{B}_{1}, \mathrm{~B}_{2}\right\}$ with $\mathrm{B}_{1}=(1.03,0.49), \mathrm{B}_{2}=$ $(1.01,0.435)$; set $\mathrm{C}=\left\{\mathrm{C}_{1}, \mathrm{C}_{2}\right\}$ with $\mathrm{C}_{1}=(1.02,0.42), \mathrm{C}_{2}=$ $(1.05,0.51)$. The states of the same set exhibit the same relaxation time $\tau_{\alpha}$, i.e. $\tau_{\alpha}^{(A)} \simeq 42, \tau_{\alpha}^{(B)} \simeq 150, \tau_{\alpha}^{(C)} \simeq 1550$. The exact definition of the relaxation time will be given in Sec.3.1.

From the equilibrated configuration of each state, in parallel to the usual production runs in NVE ensemble, we also started production runs in the iso-configurational ensemble (ICE) [42]. Each ICE, henceforth labeled by $\mu$, consists in a single initial spatial configuration of all the particles, each of them starting the time evolution with 1000 random assignments of the initial velocity, as drawn from the corresponding MaxwellBoltzmann distribution of the state. We considered four ICEs for the states of the set $\mathrm{C}$ and two ICEs for the other two sets, in close agreement with other studies performed with about three times less particles than the present one [45]. The initial configurations of the ICEs were randomly taken by the set of NVE production runs. The fact that the number of ICEs is less than the number of production runs in the NVE ensemble is due to the extremely time-consuming procedure to perform the MI evaluation. Arguments will be presented in Sec.3.1 to conclude that the phase space explored by all the ICEs of every single state closely matches in size the one explored by all the 


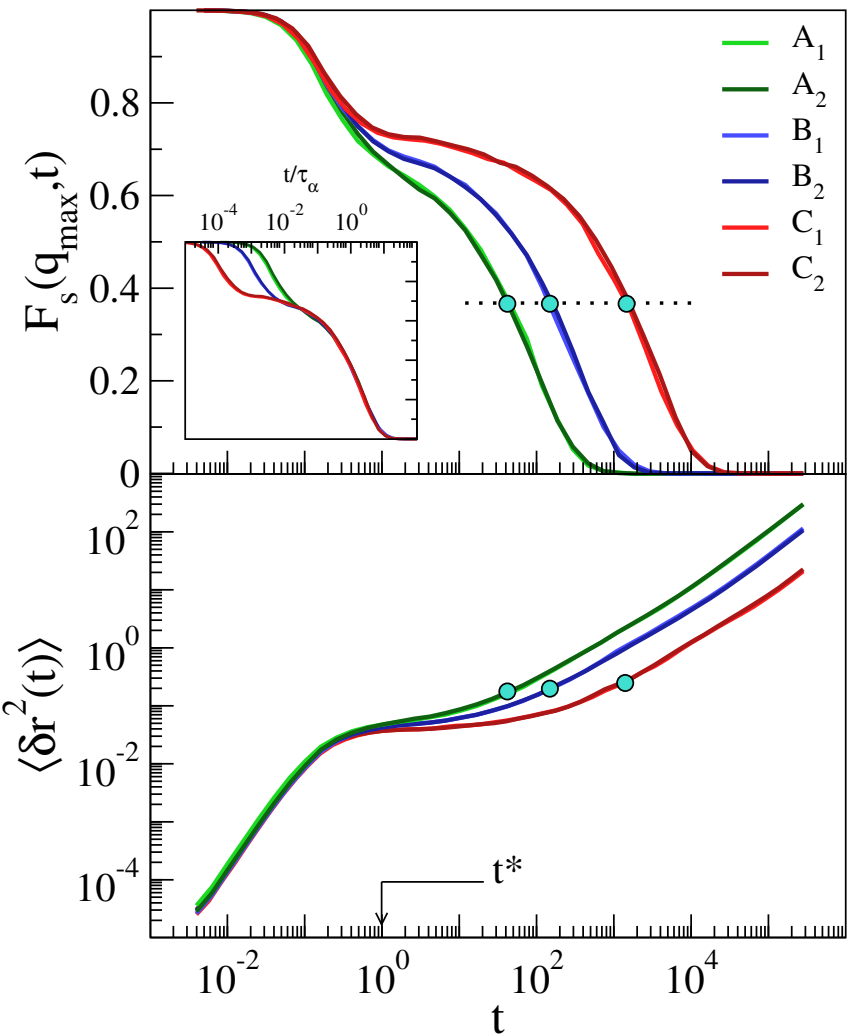

Fig. 1. Top: self-part of the intermediate scattering function (ISF) at $q_{\max }$, the fist peak of the static structure factor, of the six states of the present study. They exhibit ISFs grouped in three distinct pairs. The position of the relaxation time $\tau_{\alpha}$ of each pair is marked with a dot. Bottom: mean square displacement (MSD). States with coincident ISF exhibit coincident MSD too.

NVE runs. Following previous studies [45, 50], MI was evaluated via the Kraskov-Stögbauer-Grassberger estimator [63]. The high number of velocity assignments in each ICE ensures proper convergence of the estimator.

We point out that two different kind of averages are adopted in the present work: i) the usual ensemble average over the NVE ensemble to be denoted as $\langle X\rangle_{N V E}$. In the absence of any ambiguity the NVE subscript is understood; ii) the average $\langle X\rangle_{\text {ICEs }}$, namely the average over the different velocities and all the monomers in a single ICE followed by the average over all the ICEs, i.e. all the initial configurations. Even if the two averages yield virtually indistinguishable results in the present study within the errors, i.e. $\langle X\rangle \simeq\langle X\rangle_{I C E s}$, given the conceptual difference, it seems proper to distinguish between them.

\section{Results and discussion}

\subsection{Global relaxation and transport}

With the purpose of characterizing the dynamics in terms of mobility, we consider the mean square displacement (MSD) of a monomer with position $\mathbf{r}(t)$ at time $t$, departing from the

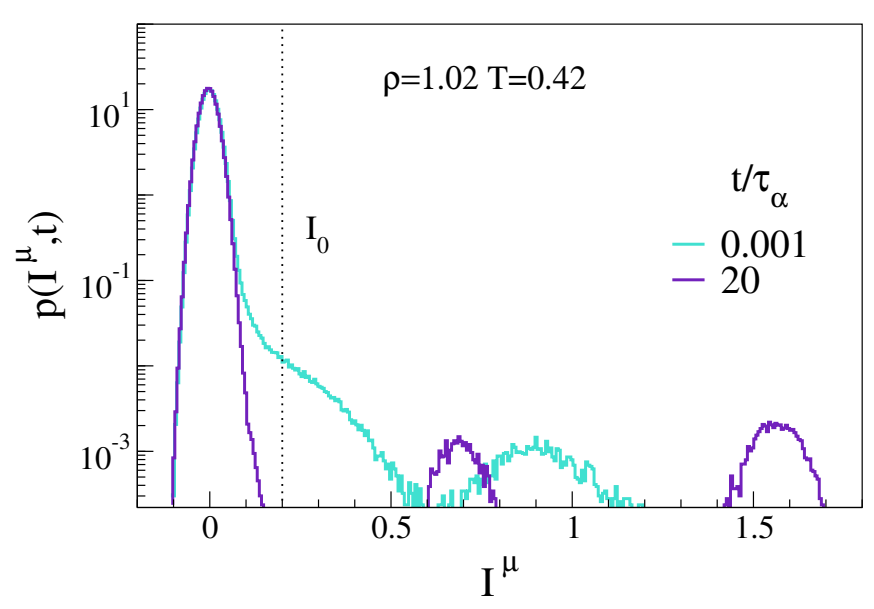

Fig. 2. Typical plots of the MI distribution at times $t$ for a single ICE of a selected state of the $\mathrm{C}$ set. The threshold $I_{0}=0.2$ above which MI values are considered significant [45] is also indicated.

initial position $\mathbf{r}(0)$ :

$\left\langle\delta r^{2}(t)\right\rangle_{k}=\frac{1}{N} \sum_{j=1}^{N}\left\langle\left\|\mathbf{r}_{j}(t)-\mathbf{r}_{j}(0)\right\|^{2}\right\rangle_{k}, k=N V E$, ICEs

Instead, relaxation is accounted for by the self-part of the intermediate scattering function (ISF):

$F_{s}^{(k)}(\mathbf{q}, t)=\frac{1}{N} \sum_{j=1}^{N}\left\langle e^{i \mathbf{q} \cdot\left[\mathbf{r}_{j}(t)-\mathbf{r}_{j}(0)\right]}\right\rangle_{k}, k=N V E, I C E s$

In an isotropic liquid ISF depends only on the modulus of the wavevector $q=\|\boldsymbol{q}\|$ and features the rearrangements of the spatial structure of the fluid over the length scale $\sim 2 \pi / q$. We define the structural relaxation time $\tau_{\alpha}$ by the relation

$F_{s}\left(q_{\max }, \tau_{\alpha}\right)=e^{-1}$ where $F_{s}(\mathbf{q}, t) \equiv F_{s}^{(N V E)}(\mathbf{q}, t)$ and $q_{\max }$ is the maximum of the static structure factor $(7.14 \leq$ $\left.q_{\max } \leq 7.22\right)$.

Fig.1 shows MSDs and ISFs of the monomers, respectively. At very short times, i.e. the ballistic regime, MSD increases according to $\left\langle r^{2}(t)\right\rangle \cong\left(3 k_{B} T / m\right) t^{2}$ and ISF starts to decay. At later times the repeated collisions with the other monomers slow down the displacement and a quasi-plateau region, also found in ISF, occurs when the temperature is lowered and/or the density increased. The plateau-like regions signal the increased trapping of the monomer in the cage of the surrounding particles. The trapped monomer is released after an average time $\tau_{\alpha}$, leading to the ISF decay and the MSD increase due to diffusive motion for $t>\tau_{\alpha}$. Owing to the choice $q=q_{\max }$, the relaxation time $\tau_{\alpha}$ corresponds to displacements of about the monomer size. Notice that $\tau_{\alpha}$ provides also a good estimate of the time needed by the trimer to diffuse by its size. In fact, the molecular size, i.e. the average end-end distance, is about 1.4 monomer size.

We define the fast mobility $\left\langle u^{2}\right\rangle$ as the MSD at $t^{*}$ [20]:

$$
\left\langle u^{2}\right\rangle=\left\langle\delta r^{2}\left(t^{*}\right)\right\rangle
$$

A proper choice of $t^{*}$ is set by the position of the inflection point of MSD seen in Fig.1 which for the present model is 
nearly constant, $t^{*} \simeq 1[20]$, corresponding to a few picoseconds [64]. Fig.1 offers, for the three pairs of states having equal FMs, an example of the correlation between the FM and the long-time relaxation, Eq. 3 with the identification $X=I S F, M S D$ It is worth noting that the presence of the bonds plays a negligible role at $t^{*}$. In fact, the $\mathrm{C}$ set which exhibits a relaxation time $\tau_{\alpha} \simeq 1550$ has fast mobility $\left\langle u^{2}\right\rangle \simeq 0.035$. The latter is rather close to the one of the species in an atomic binary mixture with Lennard-Jones potential in states with $\tau_{\alpha} \sim 1900$, i.e. $\left\langle u^{2}\right\rangle \simeq 0.03-0.04$ [30]. We ascribe this fact to the fact that the molecules are largely deformable owing to the absence of both bending and torsional potentials in our model.

\subsection{Mutual Information}

\subsubsection{Global analysis}

Following Ref. [45], we consider MI between the vector displacements of the particle pair $(i, j)$. MI is evaluated, according to Eq.4, by the relation

$$
I_{i j}^{\mu}(t)=I\left(\delta \boldsymbol{r}_{i}^{\mu}(t), \delta \boldsymbol{r}_{j}^{\mu}(t)\right)
$$

where $\delta \boldsymbol{r}_{i}^{\mu}(t)$ is the displacement of the $i$-th particle in a time $t$ starting from the initial position in the selected ICE (labeled by $\mu$ ). The particles $i$ and $j$ are said to be correlated at time $t$ if $I_{i j}^{\mu}(t)>I_{0}$ with $I_{0}=0.2$. The threshold $I_{0}$ has been chosen in agreement with other studies [45] to filter out the Gaussian noise of the estimator leading to spurious effects at small MI values. A quantity of interest is the probability distribution $p\left(I^{\mu}, t\right)$ of the MI values at time $t$ for a single ICE of the liquid. $p\left(I^{\mu}, t\right) d I^{\mu}$ is the probability that the MI between two particles at time $t$ is located in the range $\left[I^{\mu}, I^{\mu}+d I^{\mu}\right]$.

Fig. 2 presents typical plots of the distribution $p\left(I^{\mu}, t\right)$ of the MI values at time $t$ for a single ICE of the liquid. It shows that, for $t \ll \tau_{\alpha}$ the distribution $p\left(I^{\mu}, t\right)$ exhibits a disjoint structure above $I_{0}$ which develops as two well separated peaks at $t \gg \tau_{\alpha}$. The disjoint structure of $p\left(I^{\mu}, t\right)$ is missing in atomic liquids [45] and follows from the permanent bonds of the trimer linking the monomers to each other.

We define the number of correlated particles with the ith central particle for a given ICE at time $t$ as $n_{i}^{\mu}(t)$. Henceforth, $p(n, t)$ denotes the probability, evaluated by considering all ICEs, that a generic central particle is MI-correlated with $n$ surrounding ones at time $t$.

Fig. 3 plots the evolution of the distribution $p(n, t)$, for the same state considered in Fig.2. In a time $t \sim 1$, corresponding to the reduced time $t / \tau_{\alpha} \sim 7 \cdot 10^{-4}$, the monomer hits the surrounding cage a few times [43]. The collisions trigger MI-correlations with a rather limited number of particles and $p(n, t)$ peaks at very small $n$ values. As time goes by, the distribution displaces at larger $n$ values for $t / \tau_{\alpha}<1$ due to the rapid exploration of the cage by the central particle establishing MI-correlations with a larger number of surrounding neighbours. For $t / \tau_{\alpha}>1$, following the cage disappearance and the larger displacements (see Fig.1), the MI-correlated particles decrease. For $t / \tau_{\alpha} \sim 4$ the distribution exhibits a notable change of the shape with clear bimodal structure. In particular a component peaking at $n \sim 12$ is observed, revealing the

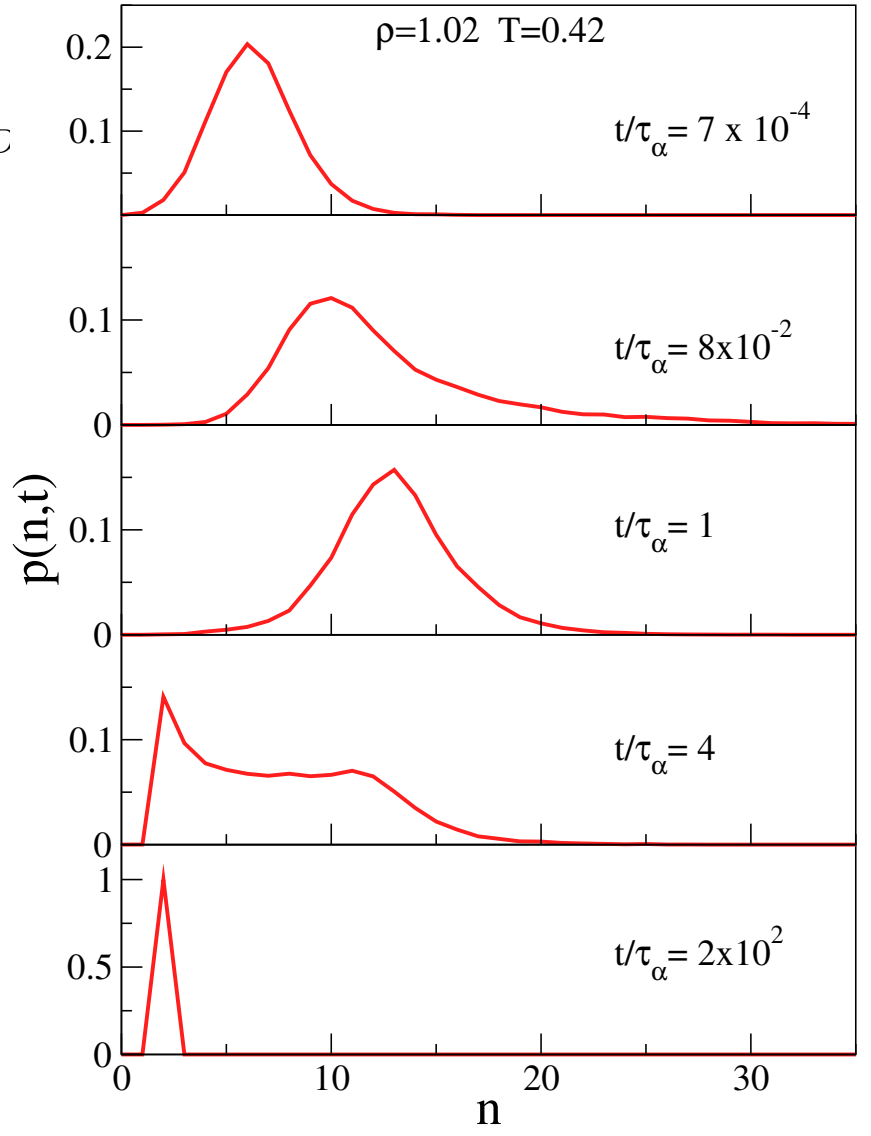

Fig. 3. Probability that a central particle is MI-correlated with $n$ surrounding ones at time $t$ for the state in Fig. 2 with relaxation time $\tau_{\alpha}^{(C)} \sim 1550$. The probability is averaged over all ICEs.

presence of monomers with persistent MI correlations. A similar bimodal pattern has been also reported in atomic liquids at the same reduced time, $t / \tau_{\alpha}=4$ [45]. However the bimodal patterns of the atomic and the molecular liquids show an important difference in the location of the peak at low $n$ values. In atomic liquid, the peak is centred at $n=0$, corresponding to the fact that at times exceeding $\tau_{\alpha}$ an increasing fraction of atoms with no MI correlations with other atoms develops, whereas in the present study, dealing with a molecular liquid of trimers, the peak is centred at $n=2$ corresponding to the fact that in a trimer each monomer has permanent correlations with two other ones. For $t \gg \tau_{\alpha}$ the distribution narrows and only the peak at about $n \sim 2$ is apparent.

Fig. 4 plots two characteristic parameters of the distribution $p(n, t)$, i.e. the average $\bar{n}(t)$ (top panel) and the standard deviation $\sigma(t)$ (bottom panel). The average number of particles which are MI-correlated with a generic one offers a measure of the average size of correlated regions at time $t$, whereas the standard deviation suggests how broad the size distribution is. It is seen that the average $\bar{n}(t)$ vanishes at short times, peaks at times $t \sim \tau_{\alpha}$ and reaches at long time the plateau level 2, already interepreted in the discussion of Fig.3. In atomic liquids $\bar{n}(t)$ vanish at long times [45]. The standard deviation $\sigma(t)$ peaks at two different times indicating the presence of two characteristic time scales other than $\tau_{\alpha}$. We refer to them 


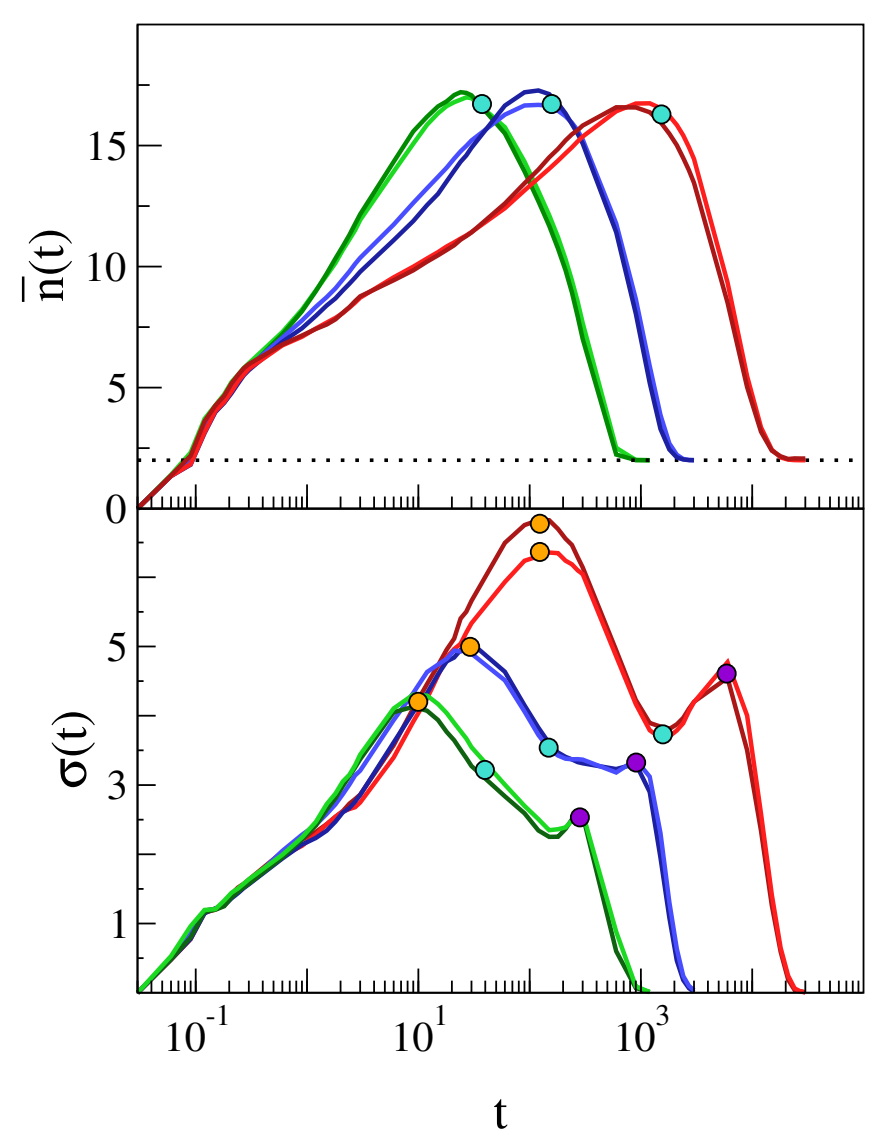

Fig. 4. Time-dependence of $\bar{n}(t)$, the average number of particles which are MI-correlated with a generic one (top), and $\sigma(t)$, the standard deviation (bottom). Color codes as in Fig.1. Note that the pairs of states belonging to the same set A, B, C exhibit the same behavior of both $n(t)$ and $\sigma(t)$. Dots mark the position of $\tau_{\alpha}$. The two peaks of the standard deviation, also reported in atomic liquids [45], identify two time scales which are referred to as $\tau_{\text {early }}$ (orange dot) and $\tau_{\text {late }}$ (violet dot).

as $\tau_{\text {early }}$ and $\tau_{\text {late }}\left(\tau_{\text {late }}>\tau_{\alpha}>\tau_{\text {early }}\right)$. On increasing $\tau_{\alpha}$, these two characteristic time scales get longer but in a different way: $\tau_{\text {early }}$ gets away from $\tau_{\alpha}$ where $\tau_{\text {late }}$ approaches the latter. The complex pattern of the standard deviation is close, but not identical, to the one of atomic liquids [45]. A remarkable difference is that in our molecular liquid the peak at $\tau_{\text {early }}$ grows in height on increasing $\tau_{\alpha}$ and the ratio between the heights of the peaks at $\tau_{\text {early }}$ and $\tau_{\text {late }}$ is fairly constant. Instead, in atomic liquids the peak at $\tau_{\text {early }}$ is absent for states with fast relaxation $\left(\tau_{\alpha} \lesssim 10\right)$ and grows more than the peak at $\tau_{\text {late }}$ when the relaxation slows down [45]. Fig.4 points out that the pairs of states with equal FM, and then equal relaxation time, exhibit the same dependence of $\bar{n}(t)$ and $\sigma_{n}(t)$ from very fast to slow time scales. We interpret the finding as novel support to the general validity of Eq.3. Finally, preliminary results about a melt of polymeric chains with $M=10$ shows that both the average $\bar{n}(t)$ and the standard deviation $\sigma(t)$ are virtually the same for $t \lesssim \tau_{\alpha}$. This is rather expected in linear molecules with high flexibility. For $t \gg \tau_{\alpha}$ the average tends to 9 whereas the standard deviation vanishes.

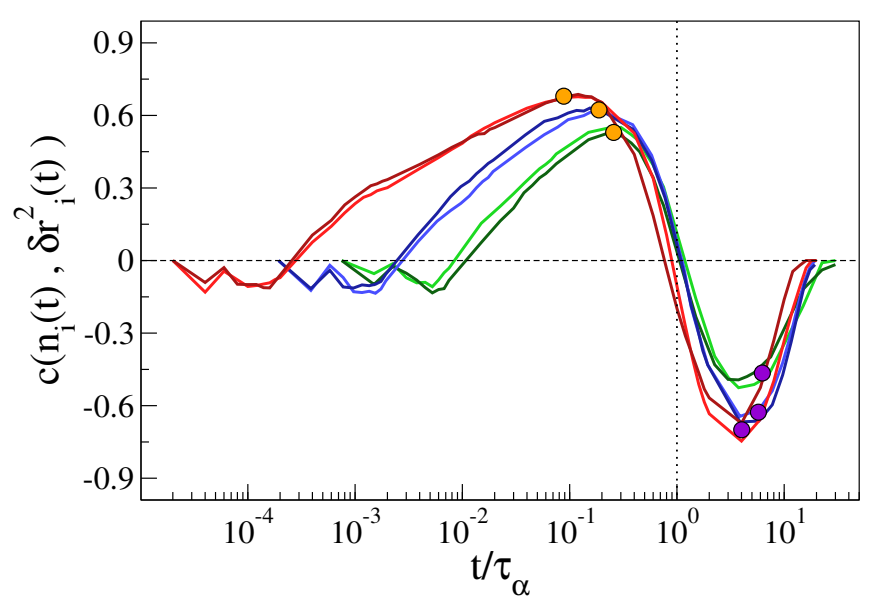

Fig. 5. Pearson correlation between the number of MI-correlated particles around the generic i-th particle at time $t$ and its propensity at the same time. Color codes as in Fig.1. Orange and violet dots mark $\tau_{\text {early }}$ and $\tau_{\text {late }}$, respectively, as defined in Fig.4 (bottom).

In order to characterize the monomers on the basis of the number of their MI-correlated particles, we consider the Pearson correlation coefficient $c\left(n_{i}(t), X\right)$, where $n_{i}(t)$ and $X$ are the number of MI-correlated particles with the generic i-th monomer at time $t$ and $X$ is a quantity of interest. Beyond an average over all the monomers, the coefficients are also averaged over all the initial configurations.

First, we examine the Pearson correlation between $n_{i}(t)$ and the propensity at the same time, i.e. the square displacement of the i-th particle in a time $t$ starting from the initial configuration. Fig.5 plots the related time evolution. It is seen that the Pearson correlation is maximum at $t \approx \tau_{\text {early }}$ and minimum at $t \approx \tau_{\text {late }}$. This clearly indicates that the monomers with higher number of surrounding MI-correlated particles than the average have higher mobility than the average at short times $\left(t<\tau_{\alpha}\right)$ and lower mobility at longer times. This aspect is substantiated in the next section. Notably, the time dependence of the correlation coefficient is the same in each pair of states with the same FM and relaxation time in agreement with Eq.3.

\subsubsection{Early-relaxing and late-relaxing fractions}

Fig.6 presents, for the same ICE of the state shown in fig.2, the correlation plot of the number of correlated particles surrounding the $\mathrm{i}$-th particle at times $\tau_{\text {early }}$ and $\tau_{\text {late }}, n_{i}^{\mu}\left(\tau_{\text {early }}\right)$ and $n_{i}^{\mu}\left(\tau_{\text {late }}\right)$ for all the monomers $i=1, \cdots, N$. A similar pattern is found in atomic liquids [45] and, in close analogy, we are led to focus on the two fractions of monomers exhibiting the largest number of MI-correlated particles. More precisely, the two fractions are formed by the monomers with a number of correlated particles exceeding the average value of $n_{i}^{\mu}\left(\tau_{\text {early }}\right)$ and $n_{i}^{\mu}\left(\tau_{\text {late }}\right)$ by two times the standard deviation. They will be referred to as early- and late-relaxing particles, respectively. Early-relaxing particles exhibit high number of correlated partners at $t=\tau_{\text {early }}$ decreasing at the longer time $\tau_{\text {late }}$. With rare exceptions, late-relaxing particles do the opposite, i.e. they exhibit a low number of correlated partners at $t=\tau_{\text {early }}$ increasing at $\tau_{\text {late. }}$. 
Fig.7 characterizes the early-relaxing and late-relaxing monomers for the two states with slower relaxation (set C). Similar features are seen for the A and B groups of states. The top and bottom panel of Fig. 7 show ISFs and MSDs, respectively, restricted to the early- and late-relaxing populations and compare them to the global curves considering all the monomers. It is seen that early-relaxing particles exhibit faster relaxation and higher mobility than the global system whereas the opposite conclusion is reached for late-relaxing particles. In particular, as conjectured in ref. [45], the restricted ISFs curves show that $\tau_{\text {early }}$ and $\tau_{\text {late }}$ are just the relaxation times of the early-relaxing and late-relaxing populations. Note that for very long times, $t \gg \tau_{\text {late }}$, the restricted MSDs merge signalling the dynamical homogenisation of the system. In agreement with Eq.3, the time dependence of the ISFs and MSDs restricted to the the early- and late-relaxing populations is found to be the same within the errors in the two states of the $\mathrm{C}$ set.

\subsubsection{Vibrational scaling of the early-relaxing and late-relaxing fractions}

We now test if the master curve Eq.2 holds for the early-relaxing and late-relaxing fractions. We remind that Eq. 2 has been originally by considering the global relaxation of a bulk system [20]. To proceed, we first specialize Eq.2 to the present model. Since the latter is characterized by $\left\langle u_{g}^{2}\right\rangle^{1 / 2}=0.129(1)$, Eq.2 is recast as [20]:

$$
\log \tau_{\alpha}=\alpha+\beta \frac{1}{\left\langle u^{2}\right\rangle}+\gamma \frac{1}{\left\langle u^{2}\right\rangle^{2}}
$$

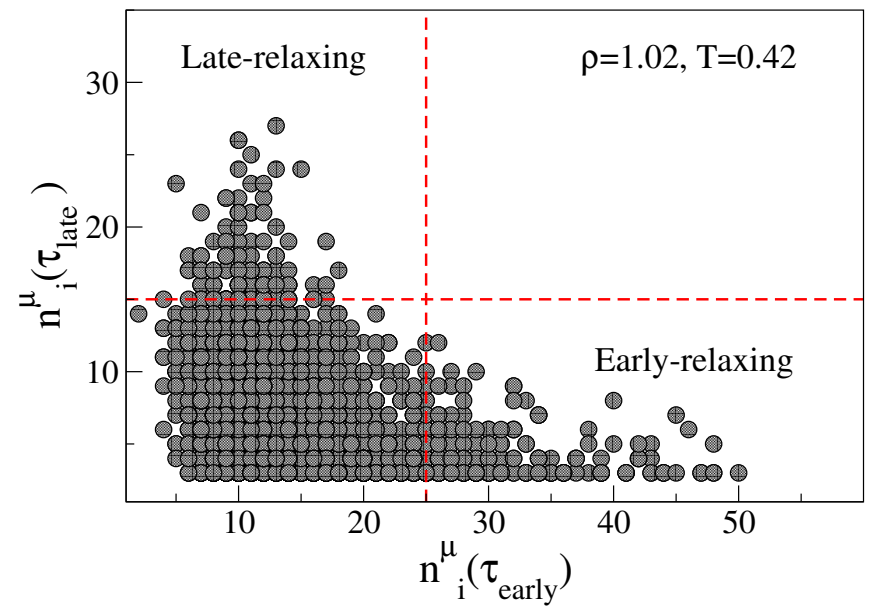

Fig. 6. Correlation plot between the number of MI-correlated particles surrounding the $\mathrm{i}$-th particle at times $\tau_{\text {early }}$ and $\tau_{\text {late }}, n_{i}^{\mu}\left(\tau_{\text {early }}\right)$ and $n_{i}^{\mu}\left(\tau_{\text {late }}\right)$. The plot includes all the monomers $i=1, \cdots, N$ in the same ICE of the state in Fig.2. The definition of $\tau_{\text {early }}$ and $\tau_{\text {late }}$ is given in Sec.3.2.1 and shown in Fig.4. The red dashed lines are positioned at the average value of $n_{i}^{\mu}(t)$ plus two times the standard deviation and divide the plot in four sectors. Particles falling in the lowerright sector and upper-left sectors are said early- and late-relaxing, respectively. When moving from $\tau_{\text {early }}$ to $\tau_{\text {late }}$, an early-relaxing particle decreases the number of MI-correlated particles, whereas - with rare exceptions - a late-relaxing particle does the opposite.

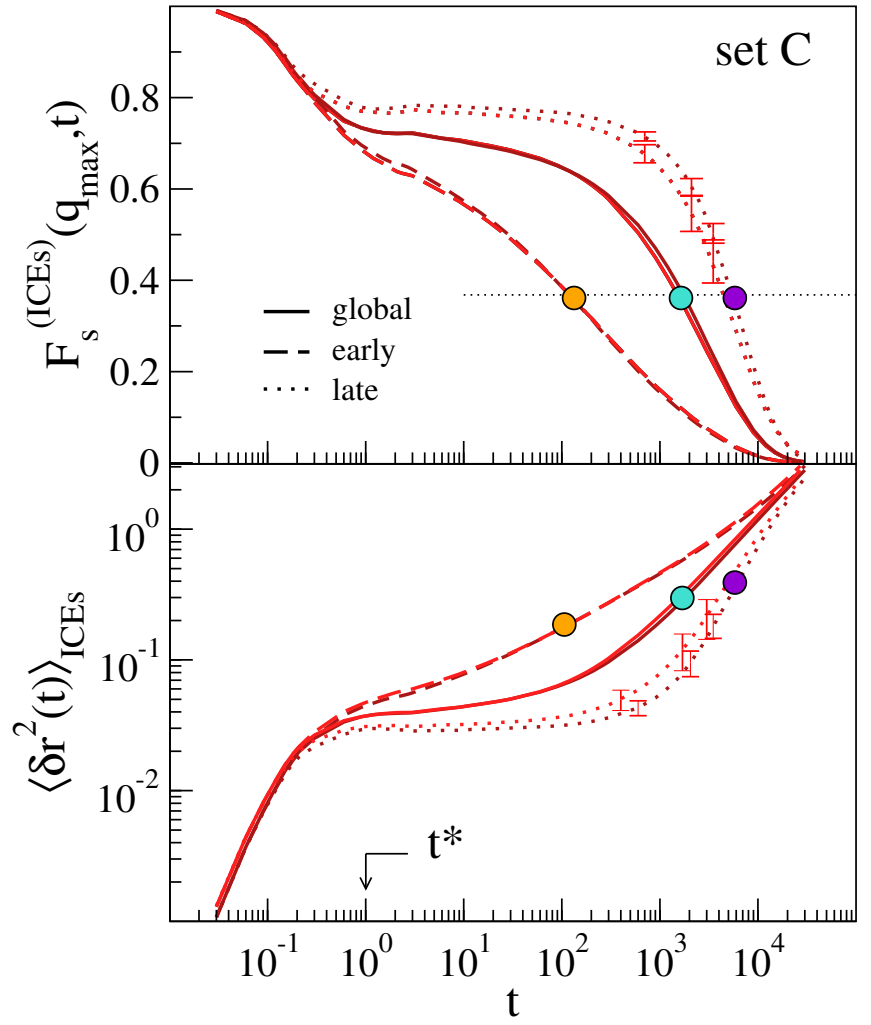

Fig. 7. Characterization of the early-relaxing and late-relaxing monomers for the two states with slower relaxation (set C). Color codes as in Fig.1. Orange and violet dots mark $\tau_{\text {early }}$ and $\tau_{\text {late }}$, respectively, as defined in Fig.4 (bottom). Top: relaxation functions by either considering all the monomers or restricting to the early-relaxing and late-relaxing monomers. The latter two functions drop to $1 / e$ at $\tau_{\text {early }}$ and $\tau_{\text {late }}$, respectively. Bottom: mean square displacements of all the system and restricted to the early-relaxing and late-relaxing monomers. The former exhibit faster mobility than the latter at intermediate times recovering a homogeneous, identical behaviour for $t \gg \tau_{\text {late. }}$. Notice that the curves referring to the same quantity are independent of the state in agreement with Eq.3.

where $\alpha=-0.424(1), \beta=2.7(1) \cdot 10^{-2}, \gamma=3.41(3) \cdot 10^{-3}$ [20]. Note that Eq.10 is written in dimensionless MD units whereas Eq. 2 is written in actual SI units.

The comparison between Eq.10 and our results are shown in Fig.8. It is seen that the vibrational scaling of the relaxation of the bulk system also holds for the the early-relaxing and late-relaxing fractions with no adjustable parameter over about three decades in relaxation time.

It is worth noting that the extension of the master curve, Eq.10, to selected fractions of a given system has been also demonstrated - with no parameter adjustment - by considering the different layers with monomer-size thickness of a molecular thin film. [37].

It is interesting to investigate the implications of the findings of Fig.8 and the related ones of ref. [37]. The model leading to Eq.10 is based on the presence of energy barriers $\Delta E \propto$ $k_{B} T a^{2} /\left\langle u^{2}\right\rangle$ where $a$ is the displacement to overcome the barrier, and $k_{B}$ is the Boltzmann constant. The usual rate theory leads to the Hall-Wolynes equation $\tau_{\alpha}^{(H W)}\left(a^{2} /\left\langle u^{2}\right\rangle\right) \propto$ 
$\exp \left(a^{2} / 2\left\langle u^{2}\right\rangle\right)$ [7]. In a disordered system one expects that the displacement $a$ is dispersed, leading to the distribution $p\left(a^{2}\right)$. In the present molecular model it is found that the distribution $p\left(a^{2}\right)$ does not change with the physical state set by the temperature, density, molecular size and non-bonding potential [20]. The mean global structural relaxation time is evaluated by averaging $\tau_{\alpha}^{(H W)}$ with $p\left(a^{2}\right)$ as weight factor [20]:

$$
\tau_{\alpha}\left(\left\langle u^{2}\right\rangle\right)=\int_{0}^{\infty} \tau_{\alpha}^{(H W)}\left(a^{2} /\left\langle u^{2}\right\rangle\right) p\left(a^{2}\right) d a^{2}
$$

Within this scheme the fact that the relation between the structural relaxation time and FM of selected parts, i.e. the "early" and "late" fractions of the present molecular liquid and the molecular layers of the thin film, is the same of the global system suggests that the distribution of the displacements to overcome the barrier is the same. Said otherwise, the parts sample effectively the global distribution $p\left(a^{2}\right)$ and all the specific details of the parts are encoded in their FM not in $p\left(a^{2}\right)$.

\subsubsection{Structure influence on the fast and the slow dynamics}

We now characterize the structure influence on the fast $\left(t<\tau_{\alpha}\right)$ and slow $\left(t<\tau_{\alpha}\right)$ dynamics. We first resort to a suitable definition of local density and, later, to a more detailed description of the spatial arrangement surrounding the particle with higher number of MI-correlated particles at short and long times.

Fig.9 presents the time evolution of the Pearson correlation between the number of MI-correlated particles with the

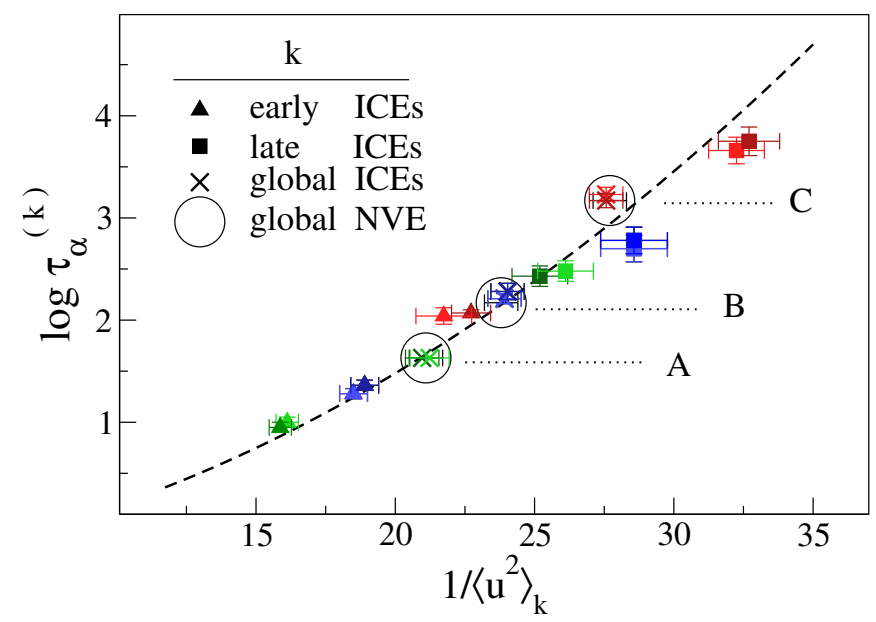

Fig. 8. Vibrational scaling of the structural relaxation time $\tau_{\alpha}^{(k)}$ vs. FM $\left\langle u^{2}\right\rangle_{k}$ for the states of the A, B and C sets. Color codes as in Fig.1. The figure shows that the scaling, originally found by considering the global relaxation, $\mathrm{k}=\{$ global, NVE $\}$, [20] and leading to the master curve Eq.10 (dashed line) [20], also applies, with no adjustable parameter, to the early- and the late-relaxing fractions, $\mathrm{k}=\{$ early, ICEs $\}$ and $\mathrm{k}=\{$ late, ICEs $\}$, respectively. Error bars are affected by the limited size of the early- and late-relaxing fractions (about $6 \%$ and $2 \%$ of the total population of particles, respectively) The empty circles enclose the global NVE averages of the three pairs of states plotted in Fig.1.

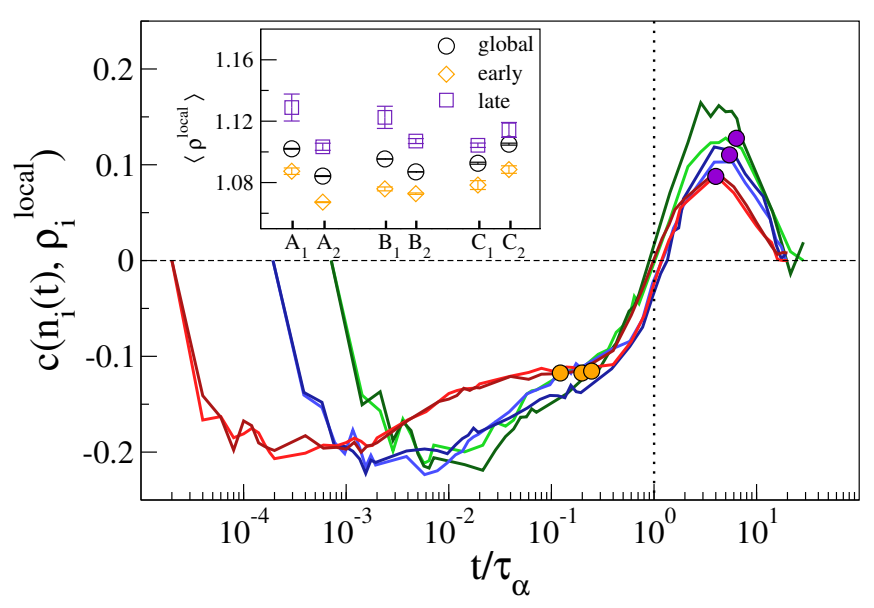

Fig. 9. Pearson correlation between the local density around the generic i-th particle in the initial configuration of the ICEs and $n_{i}(t)$, the number of MI-correlated particles at time $t$. Color codes as in Fig.1. Orange and violet dots mark $\tau_{\text {early }}$ and $\tau_{\text {late }}$, respectively, as defined in Fig.4 (bottom). For $t<\tau_{\alpha}$ particles with higher MIcorrelation belong to sites with initial lower density. For $t>\tau_{\alpha}$ particles with higher MI-correlation belong to sites with initial higher density. Inset: comparison between the density and the average local density at the sites where the early and late-relaxing particles are located for all the six investigated states. Late-relaxing monomers are located in denser sites than early-relaxing ones.

generic i-th central one at time $t$ and the local density of its surroundings at the initial time, $\rho_{i}^{\text {local }}$. The latter quantity is evaluated in a sphere of radius $r_{0}=1.45$ centred on the i-th particle. The quantity $r_{0}$ is about the position of the first minimum of the radial distribution function (virtually independent of the state) to restrict the evaluation to the first shell around the tagged particle. We see that states belonging to the same set $(A, B, C)$ exhibit the same time dependence of the correlation. The time dependence is similar to the one observed in atomic liquids [45]. For $t<\tau_{\alpha}$ a negative correlation is observed which indicates a slight tendency for the early-relaxing particles to be located in regions with lower local density. We notice that the negative correlations start to decrease in modulus at $t \simeq \tau_{\text {early }}$ and vanish very close to $\tau_{\alpha}$. For $t>\tau_{\alpha}$ the correlation is positive suggesting that late-relaxing particles tend to be located in regions with higher local density with respect to the bulk (however note that the correlations are evaluated considering all the particles). The positive correlations start to decrease at $t \simeq \tau_{\text {late }}$. As also suggested by Fig.7, Fig.9 supports the conclusion that $\tau_{\text {early }}$ and $\tau_{\text {late }}$, the times where the standard deviations of the number of correlated particles is maximum (Fig. 4), are to be interpreted as the lifetimes of the early- and late-relaxing fractions, respectively. On increasing the relaxation time $\tau_{\alpha}$ by moving from the A set of states to the C set, Fig. 9 clarifies that: i) the width of the region with negative correlation widens (in units of $\tau_{\alpha}$ ) whereas the region with positive correlations does not, ii) the magnitude of both the positive and the negative correlations do not change appreciably. The region with negative Pearson correlation between particles with high MI-correlations and local density is expected to be contributed by early-relaxing particles whereas late-relaxing particles should come up with positive Pearson correlations. In 


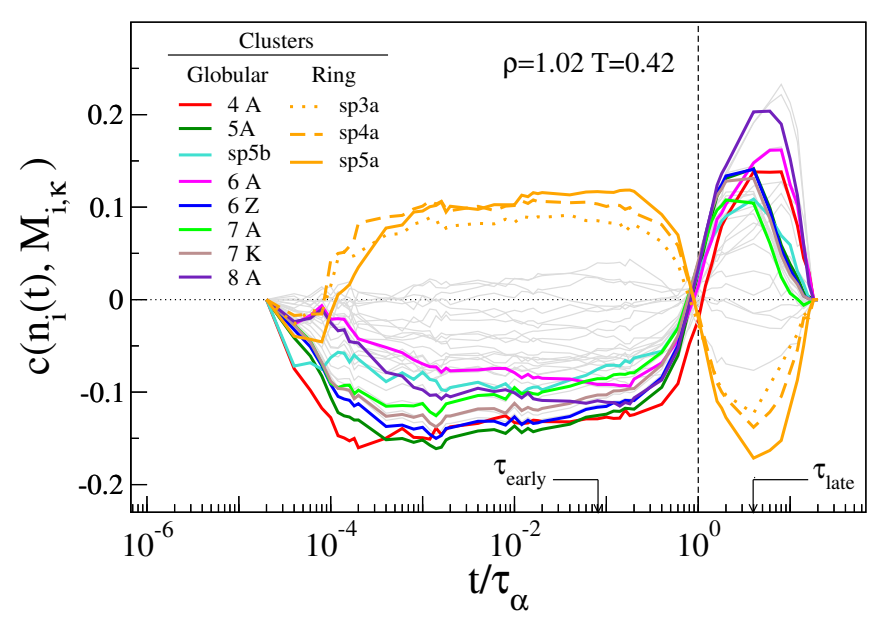

Fig. 10. Pearson correlation between $n_{i}(t)$, the number of MIcorrelated particles with the generic i-th particle at time $t$, and the number of clusters with $\kappa$ identifier owning the i-th particle in the initial configuration, $M_{i, \kappa}$. The analysis is performed for a state of the $\mathrm{C}$ set. The highlighted curves correspond to the set of clusters which maximise the modulus of the Pearson correlation at both $\tau_{\text {early }}$ and $\tau_{\text {late }}$ in all the six states of the sets A, B, C. Their $\kappa$ identifiers, according to the taxonomy of refs. [65,66], are listed in the legend. The procedure reveals correlation with ring structures at short times whereas at long times, among others, globular structures like the tetrahedron (4 A), the triangular bi-pyramid (5 A), the octahedron (6 A) and the pentagonal bi-pyramid (7 A) are signalled. The grey curves indicate the presence of several other kinds of clusters with some Pearson correlation.

fact, the inset of Fig.9 shows that the average local density of the early-relaxing particles is lower than the global one and the opposite holds true for the late-relaxing ones.

On increasing the relaxation time moving from the A set of states to the $\mathrm{C}$ set, the deviations of the local densities from the global one tend to decrease. This suggests that packing effects alone are unable to provide insight into more subtle details as the relative shift of $\tau_{\text {early }}$ and $\tau_{\text {late }}$ with respect to $\tau_{\alpha}$ noted in Fig.4. In that respect, we now investigate the role of the kind of spatial arrangements of the correlated particles.

To proceed, we employ the topological cluster classification (TCC) of the local structure [66] being adopted to investigate the structure of a large variety of system, ranging from simple liquid to colloid-polymer mixtures [67-69]. TCC relies on a modified Voronoi decomposition to identify the neighbourhood topology and compare the latter with the one of isolated equilibrated clusters. In particular, we analyse the structure of the initial spatial configuration and identify the topological clusters owning each particle. We remind that a particle can be owned by more than one cluster, even of the same kind.

Fig.10 shows the Pearson correlation between the generic i-th particle with $n_{i}(t)$ MI-correlated surrounding particles at time $t$ and the number of different kind of clusters labelled by the $\kappa$ identifier owning the $\mathrm{i}$-th particle in the initial configuration, $M_{i, \kappa}$ for a state of the $\mathrm{C}$ set. The complete list of identifiers and the corresponding description of the clusters is given elsewhere $[65,66]$. The high numbers of curves signals that the kinds of cluster with some Pearson correlation is not low. In order to devise a viable procedure to compare the six

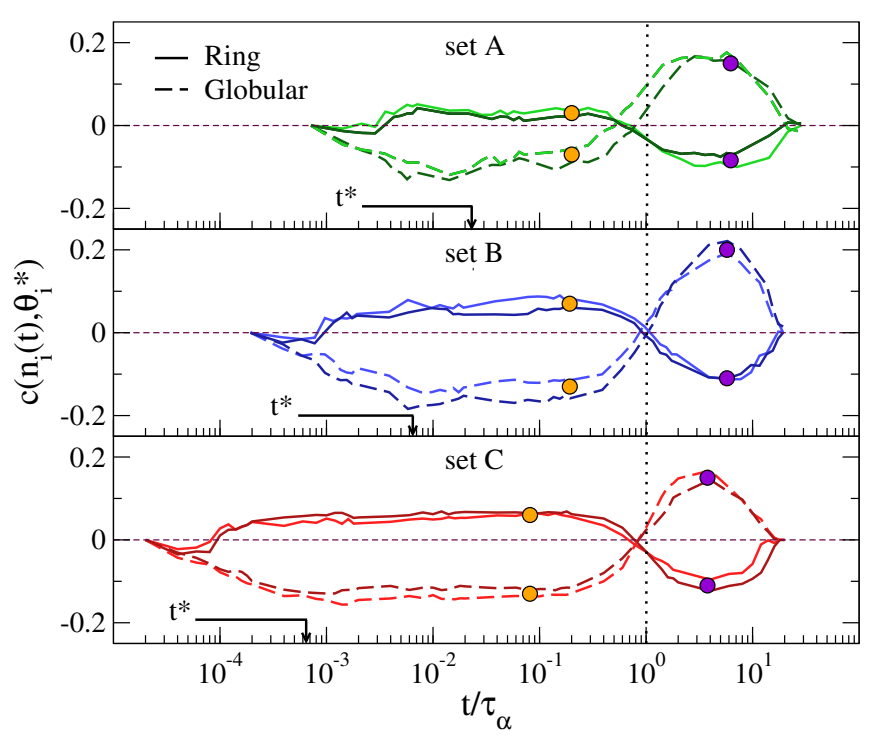

Fig. 11. Pearson correlation between $n_{i}(t)$, the number of MIcorrelated particles with the generic i-th particle at time $t$, and the number of either ring (planar) or globular clusters identified in Fig.10 owning the i-th particle in the initial configuration, $M_{i, R}$ and $M_{i, G}$, respectively. Color codes as in Fig.1. Orange and violet dots mark $\tau_{\text {early }}$ and $\tau_{\text {late }}$ (violet dot), respectively.

states forming the A, B and C sets, we highlighted the kind of clusters which maximise the modulus of the Pearson correlation at both $\tau_{\text {early }}$ and $\tau_{\text {late }}$ in all the six states. These clusters are believed to be better correlated to the dynamics in a state-independent way. Fig.10 shows that for $t<\tau_{\alpha}$ particles with higher number of MI-correlated particles than the average belong to quasi-planar clusters of 3, 4 and 5 particles known as shortest path ring which are associated to lower local density [45]. This agrees with Fig.9. Differently, for $t>\tau_{\alpha}$ highly correlated particles belong to globular clusters like the tetrahedron $(4 \mathrm{~A})$, the triangular bi-pyramid $(5 \mathrm{~A})$, the octahedron $(6$ A), the pentagonal bi-pyramid (7 A) and other complex structures with higher number of particles [66].

It must be pointed out that some of our selected clusters are also revealed in atomic liquids [45]. As an example, ring clusters sp3, sp4 and sp5, associated to the the early-relaxing population. Instead, globular clusters like 10B and 12D, detected in the late-relaxing population of atomic liquids do not pass our selection criteria owing to low Pearson correlation. Moreover, long long-lived clusters in hard sphere systems like cosahedron (13A) [70] are barely detected in our molecular system.

Even if the above selection procedure captures significant structural features of the clusters owning the particles with high MI correlation, it still exhibits some small state-dependence in the sense that the magnitude of the Pearson correlation of the selected clusters still depends on the state. In order to reduce this effect, we consider the Pearson correlation between $n_{i}(t)$, the number of MI-correlated particles with the generic i-th particle at time $t$, and the number of either ring (planar) or globular clusters identified in Fig.10 owning the i-th particle in the initial configuration, $M_{i, R}$ and $M_{i, G}$, respectively. The results are shown in Fig.11. Interestingly, this less detailed characterization offers additional insight. As to the the relative shift of 
$\tau_{\text {early }}$ and $\tau_{\text {late }}$ with respect to $\tau_{\alpha}$ noted in Fig.4, one sees that $\tau_{\text {early }}$ and $\tau_{\text {late }}$ provide not only a measure of the relaxation time of the the early-relaxing and the late-relaxing fractions, but they also offer a measure of the lifetime of selected subgroups, i.e the ring and the globular clusters respectively. Furthermore, Fig. 11 recovers the property, Eq.3, i.e. states with the same FM exhibit rather close time dependence at all times from vibrational time scales to long times of a specific quantity, i.e. the Pearson correlation (less apparent in the B set) between particles with high number of MI-correlated particles and the number of their inclusions in specific sets of either planar or globular clusters. This finding suggests that Eq.3, at least in part, is enforced by structure. A similar indication was reached by considering the scaling between elasticity and relaxation [41].

The validity of Eq.3 shown in Fig. 11 is in harmony with several other quantities presented in this study, i.e. global ISF and MSD (fig.1), average and standard deviation of the global distribution of MI-correlated monomers (fig.4), Pearson correlation coefficients of number of MI-correlated particles with propensity (fig.5) and local density (fig.9). All in all, these findings further corroborate our previous conclusion that physical states with identical vibrational properties, as quantified by FM, manifest strong dynamic similarity at long times, i.e at least the structural relaxation time [20-37].

\section{Conclusions}

The heterogeneous dynamics in a mildly supercooled molecular liquid has been investigated by MD simulations. The analysis revealed MI correlation between propensities which allowed the identification of two particle fractions with different mobility and relaxation. The two fractions exhibit vibrational scaling of their relaxation with master curve not differing from the one found for the bulk, thus confirming identical results found in other systems with strong dynamic heterogeneity as thin molecular films. Packing effects are unable to clarify finer aspects of the dynamics of the two fractions. A more refined analysis suggests excitation of planar and globular structures at short and long times, respectively. The observation that states with equal fast mobility have identical time dependence of any other quantity considering all the particles, at least up to $\tau_{\alpha}$, has been extended to quantities restricted to the fractions with heterogeneous dynamics.

We acknowledge the support from the project PRA-2018-34 ("ANISE") from the University of Pisa. A generous grant of computing time from IT Center, University of Pisa and Dell EMC ${ }^{\circledR}$ Italia is also gratefully acknowledged.

\section{Authors contributions}

All the authors were involved in the preparation of the manuscript. All the authors have read and approved the final manuscript.

\section{References}

1. P.G. Debenedetti, Metastable Liquids (Princeton University Press, Princeton USA, 1997)
2. L. Berthier, G. Biroli, Rev. Mod. Phys. 83, 587 (2011)

3. M.D. Ediger, P. Harrowell, J. Chem. Phys. 137, 080901 (2012)

4. C.A. Angell, K.L. Ngai, G.B. McKenna, P. McMillan, S.W.Martin, J. Appl. Phys. 88, 3113 (2000)

5. R. Richert, J. Phys.: Condens. Matter 14, R703 (2002)

6. A. Tobolsky, R.E. Powell, H. Eyring, Elastic-viscous properties of matter, in Frontiers in Chemistry, edited by R.E. Burk, O. Grummit (Interscience, New York, 1943), Vol. 1, pp. 125-190

7. R.W. Hall, P.G. Wolynes, J. Chem. Phys. 86, 2943 (1987)

8. C.A. Angell, Science 267, 1924 (1995)

9. J.C. Dyre, N.B. Olsen, T. Christensen, Phys. Rev. B 53, 2171 (1996)

10. L.M. Martinez, C.A. Angell, Nature 410, 663 (2001)

11. K.L. Ngai, Phil. Mag. 84, 1341 (2004)

12. K.L. Ngai, J. Non-Cryst. Solids 275, 7 (2000)

13. X. Xia, P.G. Wolynes, Proc. Natl. Acad. Sci. USA 97, 2990 (2000)

14. U. Buchenau, R. Zorn, Europhys. Lett. 18, 523 (1992)

15. F. Starr, S. Sastry, J.F. Douglas, S. Glotzer, Phys. Rev. Lett. 89, 125501 (2002)

16. P. Bordat, F. Affouard, M. Descamps, K.L. Ngai, Phys. Rev. Lett. 93, 105502 (2004)

17. A. Widmer-Cooper, P. Harrowell, Phys. Rev. Lett. 96, 185701 (2006)

18. H. Zhang, D.J. Srolovitz, J.F. Douglas, J.A. Warren, Proc. Natl. Acad. Sci. USA 106, 7735 (2009)

19. A. Widmer-Cooper, H. Perry, P. Harrowell, D.R. Reichman, Nature Physics 4, 711 (2008)

20. L. Larini, A. Ottochian, C. De Michele, D. Leporini, Nature Physics 4, 42 (2008)

21. A. Ottochian, C. De Michele, D. Leporini, J. Chem. Phys. 131, 224517 (2009)

22. F. Puosi, D. Leporini, J.Phys. Chem. B 115, 14046 (2011)

23. A. Ottochian, D. Leporini, Philosophical Magazine 91, 1786 (2011)

24. A. Ottochian, D. Leporini, J. Non-Cryst. Solids 357, 298 (2011)

25. C. De Michele, E. Del Gado, D. Leporini, Soft Matter 7, 4025 (2011)

26. F. Puosi, D. Leporini, J. Chem. Phys. 136, 211101 (2012)

27. F. Puosi, D. Leporini, J. Chem. Phys. 136, 164901 (2012)

28. F. Puosi, D. Leporini, J. Chem. Phys. 139, 029901 (2013)

29. D.S. Simmons, M.T. Cicerone, Q. Zhong, M. Tyagic, J.F. Douglas, Soft Matter 8, 11455 (2012)

30. F. Puosi, C.D. Michele, D. Leporini, J. Chem. Phys. 138, 12 A532 (2013)

31. A. Ottochian, F. Puosi, C.D. Michele, D. Leporini, Soft Matter 9, $7890(2013)$

32. V.N. Novikov, A.P. Sokolov, Phys. Rev. Lett. 110, 065701 (2013)

33. F. Puosi, O. Chulkin, S. Bernini, S. Capaccioli, D. Leporini, J. Chem. Phys. 145, 234904 (2016)

34. E. Guillaud, L. Joly, D. de Ligny, S. Merabia, The Journal of Chemical Physics 147, 014504 (2017)

35. R. Horstmann, M. Vogel, The Journal of Chemical Physics 147, 034505 (2017)

36. F. Puosi, D. Leporini, J. Chem. Phys. 148, 131102 (2018)

37. M. Becchi, A. Giuntoli, D. Leporini, Soft Matter 14, 8814 (2018)

38. B.A. Pazmiño Betancourt, P.Z. Hanakata, F.W. Starr, J.F. Douglas, Proc. Natl. Acad. Sci. USA 112, 2966 (2015)

39. C. Angell, J.Non-Crystalline Sol. 131-133, 13 (1991)

40. J.F. Douglas, B.A. Pazmiño Betancourt, X. Tong, H. Zhang, J. Stat. Mech.: Theory Exp. p. 054048 (2016)

41. F. Puosi, D. Leporini, J. Chem. Phys. 136, 041104 (2012) 
42. A. Widmer-Cooper, P. Harrowell, H. Fynewever, Phys. Rev. Lett. 93, 135701 (2004)

43. S. Bernini, D. Leporini, The Journal of Chemical Physics 144, 144505 (2016)

44. W. Li, Journal of Statistical Physics 60, 823 (1990)

45. A.J. Dunleavy, K. Wiesner, R. Yamamoto, C.P. Royall, Nature Communications 6, 6089 (2015)

46. J. Iaconis, S. Inglis, A.B. Kallin, R.G. Melko, Physical Review B 87, 195134 (2013)

47. P.V. Sriluckshmy, I. Mandal, Journal of Statistical Mechanics: Theory and Experiment 2018, 043301 (2018)

48. M.C. Gao, M. Widom, The Journal of Physical Chemistry B 122, 3550 (2018)

49. A.J. Dunleavy, K. Wiesner, C.P. Royall, Phys. Rev. E 86, 041505 (2012)

50. R.L. Jack, A.J. Dunleavy, C.P. Royall, Phys. Rev. Lett. 113, 095703 (2014)

51. S. Plimpton, J. Comput. Phys. 117, 1 (1995)

52. http://lammps.sandia.gov

53. M. Doi, S.F. Edwards, The Theory of Polymer Dynamics (Clarendon Press, 1988)

54. D. Prevosto, S. Capaccioli, M. Lucchesi, D. Leporini, P. Rolla, J. Phys.: Condens. Matter 16, 6597 (2004)

55. A. Barbieri, G. Gorini, D. Leporini, Phys. Rev. E 69, 061509 (2004)

56. A. Barbieri, E. Campani, S. Capaccioli, D. Leporini, J. Chem. Phys. 120, 437 (2004)

57. L. Andreozzi, M. Faetti, M. Giordano, D. Leporini, The Journal of Physical Chemistry B 103, 4097 (1999)

58. L. Andreozzi, M. Faetti, M. Giordano, D. Leporini, J.Phys.:Condens. Matter 11, A131 (1999)

59. L. Andreozzi, M. Giordano, D. Leporini, J. Non-Cryst. Solids 235, 219 (1998)

60. D. Leporini, Phys. Rev. A 49, 992 (1994)

61. M. Giordano, D. Leporini, M. Martinelli, L. Pardi, S. Santucci, C. Umeton, The Journal of Chemical Physics 88, 607 (1988)

62. L. Larini, R. Mannella, D. Leporini, The Journal of Chemical Physics 126, 104101 (2007)

63. A. Kraskov, H. Stögbauer, P. Grassberger, Phys. Rev. E 69 , 066138 (2004)

64. M. Kröger, Phys. Rep. 390, 453 (2004)

65. J.P.K. Doye, D.J. Wales, R.S. Berry, J. Chem. Phys. 103, 4234 (1995)

66. A. Malins, S.R. Williams, J. Eggers, C.P. Royall, The Journal of Chemical Physics 139, 234506 (2013)

67. J. Taffs, A. Malins, S.R. Williams, C.P. Royall, J. Phys.: Condens. Matter 22, 104119 (2010)

68. C.P. Royall, S.R. Williams, T. Ohtsuka, H. Tanaka, Nat. Mater. 7, $556(2008)$

69. C.P. Royall, S.R. Williams, The Journal of Physical Chemistry B 115, 7288 (2011)

70. C.P. Royall, A. Malins, A.J. Dunleavy, R. Pinney, Journal of NonCrystalline Solids 407, 34 (2015) 